\title{
On the Gaussian Fading Broadcast Relay Channel with Causal State Feedback
}

\author{
Chao He, Student Member, IEEE, Sheng Yang, Member, IEEE, and Pablo Piantanida, Member, IEEE
}

\begin{abstract}
We investigate a typical setup of heterogeneous network that can be modeled as a fast Rayleigh fading broadcast relay channel (BRC) with one source (macrocell BS), one fullduplex relay (smallcell BS), and two destinations (mobile users). It is assumed that instantaneous channel state information (CSI) is available at the receivers' side while only a noiseless causal state feedback from the destinations about the source-to-destination channels is available at the relay. We propose a relaying scheme based on a two-stage decode-compress-forward (DCF) strategy.

The essential idea is to let the relay decode the source messages and then forward to the destinations a function of both the decoded messages and the CSI feedback. By characterizing and evaluating the achievable rate region of the proposed relaying scheme, we reveal that non-negligible performance gains over conventional cooperative strategies can be obtained by exploiting CSI feedback at the relay.
\end{abstract}

Index Terms-Heterogeneous network, Broadcast channel (BC), Relay channel (RC), Multiple antenna communication, State feedback

\section{INTRODUCTION}

Future wireless communication systems will feature ultra dense and heterogeneous device population. As the data traffic explodes, interference will become the bottleneck of overall network throughput. One way to accommodate the exponential growth of network data flow is to take advantage of the spatial multiplexing gain provided by multi-antenna transmission techniques (MIMO, or even massive MIMO). Another way is to exploit the spatial reuse by densifying the deployment of BSs while allowing cooperation between multiple-tier BSs via wired (distributed antenna system with backhaul) or wireless (relaying) links. Motivated by the latter, we consider a typical heterogeneous network setup in which a macrocell BS serves two users who are in close proximity to a smallcell (e.g., femto/pico) BS. A natural question to ask here is whether and how smallcell BS could improve downlink performance of the macrocell network. Essentially, the smallcell BS in this setting may be considered as a relay, and the overall channel becomes a broadcast relay channel (BRC).

Capacity characterization of general relay networks is a widely open problem, to such an extent that even the capacity of the three-node relay network is still unknown except for a few special cases (e.g., the degraded and reversely degraded

Chao He, Sheng Yang, and Pablo Piantanida are with the Laboratoire des Signaux et Systmes (L2S, UMR CNRS 8506), CNRS - CentraleSupelec - Université Paris-Sud, 3, rue Joliot-Curie, 91190, Gif-sur-Yvette, France. ( $\{$ chao.he, sheng.yang, pablo.piantanida\}@ $\}$ centralesupelec.fr).

The material in this paper was presented in part at the 2015 IEEE International Conference on Communications, June. 2015. relay channels) [1]. Nevertheless, achievability results (without the proof of optimality), also known as inner bounds of the capacity region, for different relay networks have been well established based on classical relaying strategies such as decode-forward (DF) and compress-forward (CF) (see, e.g., [2]-[4]). As for the Gaussian noise broadcast channel (without relay), capacity region is known when the channel state is deterministic [5], [6]. When the channel is subject to fading unknown to the transmitter (no CSIT), the capacity is still open except for some particular fading processes such as the case when both users experience the exact same fading distribution [7]. For more general cases, only high SNR (signal-to-noise ratio) characterizations in terms of degrees of freedom (DoF) are available in the literature. Through the DoF analysis, it is by now well acknowledged that timely and accurate CSIT is crucial for wireless networking with distributed receivers [8], [9].

In most practical scenarios, however, the available CSIT may not be accurate for various reasons, namely, noisy CSIT caused by poor feedback quality (or insufficient uplink training, e.g., for TDD systems) or outdated feedback due to high user mobility. If the CSIT quality could be improved by investing more resources at the uplink, the timeliness of the CSI feedback cannot be guaranteed anyhow when the channel varies too rapidly. Yet, it turns out that completely outdated CSIT can still be very useful, i.e., almost linear DoF gain with the number of users and the number of transmit antennas at the BS can be obtained [10]. This conclusion holds as long as the transmitter can receive the CSI feedback (with arbitrary but finite delay) from all receivers and that the CSI error vanishes as $O\left(\mathrm{SNR}^{-1}\right)$. The proposed scheme can be interpreted as the space-time interference alignment which is possible with the knowledge of the past channel at the transmitter. Since [10], several extensions have been made in various configurations (cf. [11]-[16]). The main idea of these works, also referred to as the MAT scheme, consists in two phases: 1) the broadcast phase in which signal containing new messages is sent to the receivers, and 2) the multicast phase in which side information generated based on both the past channel and the sent messages is diffused. It is therefore intuitive to think of the transmitter as two separate virtual transmitters: one takes charge of the first phase without the need of any CSIT, whereas the other is responsible for the second phase and uses the delayed CSIT and the transmitted message. The second virtual transmitter is very much like a relay that "learns" the messages and the channel coefficients and "forwards" the necessary side information to "help" the receivers decode their own messages. 
In this work, we deploy an infrastructure relay that helps accomplish the space-time interference alignment a la MAT with the feedback from every mobiles to the smallcell BS (relay) instead to the macrocell BS (source). Our setting on feedback is mainly motivated by the fact that feeding back CSI accurately to the macrocell BS via uplink is costly in practice. The cost is in terms of both the uplink bandwidth and the uplink transmit power related to the feedback. Since the relay is assumed to be closer to the destinations than the source is, feedback is to the relay instead may be more feasible. In other words, the feedback communication is localized and the feedback power could be reduced thanks to lower channel attenuation. In particular, we investigate the potential performance gain from the use of relay in the presence of CSI feedback from the destinations to the relay. We propose a decode-compressforward (DCF) relaying scheme that exploits the causal CSI feedback and the derivation of the corresponding achievable rate region. The proposed scheme is first presented for the general two-user memoryless broadcast relay channel, and then specified for the fast fading Gaussian noise channel (which is a special case of memoryless channels). Our scheme is based on the aforementioned space-time interference alignment with the help of a relay. In particular, we consider two variants of the DCF scheme according to how the source messages are sent and relayed, that is, simultaneous emitted DCF (SE-DCF) and time division DCF (TD-DCF). The main contribution of our work lies in the characterization and evaluation of the rate region achieved by the proposed schemes. With numerical simulation focusing on the Gaussian case, we compare our schemes with the conventional decode-forward (DF) and compress-forward (CF) schemes directly adapted to the fading BRC. Our results reveal that the proposed scheme provides a non-negligible gain over conventional schemes in terms of sum-rate performance, especially in the high SNR regime.

Some other related works are worth mentioning as follows. The impact of noisy CSI (statistic only/limited feedback) at BS/relay is investigated in [17], [18] on a downlink of OFDMA multi-relay cellular system in which a two-phase relaying transmission strategy is employed. In particular, it is observed that the average achievable rate performance with noisy CSIT and perfect CSIT are not comparable and yet noisy CSIT is promising within the practical scenario in the sense of exploiting relay-assisted diversity gain. In [19], a sum-rate degradation is reported in two-hop BRC that results from the low quality of CSI feedback with the assumption of quantized channel state feedback performed from users to the relay and then to the BS.

The remainder of the paper is organized as follows. Section II introduces the general BRC model and the corresponding Gaussian noise channel. Section III provides the achievable regions in general BRC while Section IV characterizes the regions by the variants of the proposed scheme in the Gaussian case. Numerical results are shown in Section V. Finally, Section VI concludes the paper. The achievability proof is presented in the appendix.

Notations: Vectors and matrices are denoted by boldface lower-case letters $\boldsymbol{a}$ and upper-case letters $A$, respectively. $A^{T}$, $\boldsymbol{A}^{H}, \operatorname{tr}(\boldsymbol{A})$ and $\operatorname{det}(\boldsymbol{A})$ are used to denote matrix transpose,
Hermitian transpose, trace and determinant, respectively. $I$ is the identity matrix. $A \succeq 0$ means that $A$ is non-negative semidefinite. We use $x^{n}$ to denote a sequence $x_{1}, x_{2}, \cdots, x_{n}$. $\mathcal{C N}(\boldsymbol{\mu}, \boldsymbol{\Sigma})$ is the circularly symmetric Gaussian distribution with mean vector $\boldsymbol{\mu}$ and covariance matrix $\boldsymbol{\Sigma}$. $\|\cdot\|$ is the Euclidean norm. $\mathbb{E}(\cdot)$ stands for the expectation operator. We use $f(x)=O(g(x))$ as the standard Landau notation. Logarithm is to the base 2 . The set of strong typical $n$-length sequences is denoted by $\mathcal{T}_{\delta}^{n}(\cdots)$ with $\delta>0$. $|\cdot|$ stands for the cardinality of the corresponding set.

\section{SySTEM MODEL}

We consider a single-cell downlink heterogeneous channel setup with one macro BS (source) and two mobile users (destinations) who are helped by a smallcell BS (relay), as shown in Fig. 1(a). The source transmits two independent messages, $W_{1}$ and $W_{2}$, to destinations 1 and 2, respectively. We assume that all the nodes share the same spectrum and the relay works in full-duplex mode, i.e., it can transmit and receive simultaneously. In this work, we focus on the fast fading scenario, i.e., the realization of the channel state at each time slot varies randomly and independently according to an identical distribution. ${ }^{1}$ Furthermore, the instantaneous channel state is unknown at the transmitters' side while it can be learned perfectly at the receivers' side at the end of each time slot.

The particularity of our model lies in the existence of an independent feedback link from the destinations to the relay, instead of to the source. This is motivated by the proximity of the relay to the destinations. At the end of each time slot, the destinations send the corresponding state information to the relay using this local communication link. For simplicity, we assume that the feedback link is noiseless and that the cost can be ignored.

To analyze such a system, we resort to an informationtheoretic framework in which a general class of memoryless broadcast relay channels are defined in the following. Based on the results obtained in such a framework, we will then focus on the Gaussian noise channel with fast fading.

\section{A. General memoryless two-user BRC}

The considered downlink channel belongs to a broader class of memoryless two-user BRC, as shown in Fig. 1(b), which is defined by the joint probability mass function (pmf)

$$
p\left(y_{1}^{n}, y_{2}^{n}, y_{r}^{n} \mid x^{n}, x_{r}^{n}, s^{n}\right)=\prod_{i=1}^{n} p\left(y_{1 i}, y_{2 i}, y_{r i} \mid x_{i}, x_{r i}, s_{i}\right)
$$

where $x^{n}$ and $x_{r}^{n}$ denote the sequence of $n$ input symbols at the source and the relay, respectively; $y_{1}^{n}, y_{2}^{n}$, and $y_{r}^{n}$ stand for the channel outputs at user 1 , user 2 , and the relay, respectively; $s^{n}$ defines the channel state. The source encoder maps each pair of messages $W_{1} \in \mathcal{W}_{1} \triangleq\left\{1,2, \cdots, 2^{n R_{1}}\right\}, W_{2} \in \mathcal{W}_{2} \triangleq$ $\left\{1,2, \cdots, 2^{n R_{2}}\right\}$ into a sequence of input symbols $x^{n}$; the

\footnotetext{
${ }^{1}$ Different fading assumptions such as the block fading can also be considered, although the interplay between the feedback delay and the coherence time should be taken into account in such settings.
} 


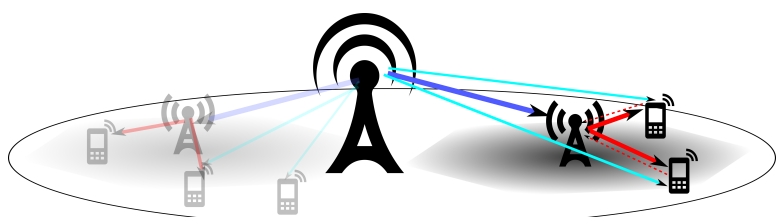

(a) Broadcast relay channel in a downlink heterogeneous network.

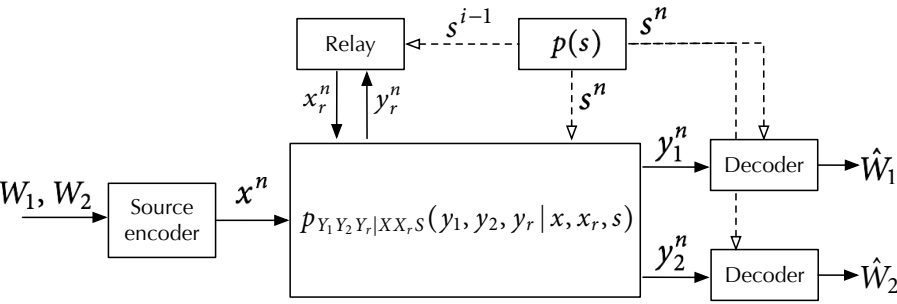

(b) General memoryless two-user broadcast relay channel.

Fig. 1. System Model: BRC in a downlink heterogeneous network and general memoryless two-user broadcast relay channel.

relay encoder maps its past observations $\left(y_{r}^{i-1}, s^{i-1}\right)$, from the source and from the destinations through feedback link, to a symbol $x_{r, i}$ at each time slot $i$; each destination $k$ decodes its own message, $\hat{W}_{k}$, from the received signal $y_{k}^{n}$ and the state sequence $s^{n}$, for $k=1,2$. A rate pair $\left(R_{1}, R_{2}\right)$ is achievable if there exists a combination of such encoding/relaying/decoding functions such that the probability of error in decoding either of the messages goes to zero with $n$.

\section{B. Fading Gaussian noise broadcast relay channel}

As a special case of the memoryless BRC, a Gaussian noise $\mathrm{BRC}$ is defined as

$$
\begin{aligned}
& \boldsymbol{y}_{1}[i]=\boldsymbol{H}_{1}[i] \boldsymbol{x}[i]+\boldsymbol{G}_{1}[i] \boldsymbol{x}_{r}[i]+\boldsymbol{z}_{1}[i], \\
& \boldsymbol{y}_{2}[i]=\boldsymbol{H}_{2}[i] \boldsymbol{x}[i]+\boldsymbol{G}_{2}[i] \boldsymbol{x}_{r}[i]+\boldsymbol{z}_{2}[i], \\
& \boldsymbol{y}_{r}[i]=\boldsymbol{H}_{r}[i] \boldsymbol{x}[i]+\boldsymbol{z}_{r}[i], \quad i=1,2, \ldots, n
\end{aligned}
$$

where $\boldsymbol{x}[i] \in \mathbb{C}^{n_{t s} \times 1}, \boldsymbol{x}_{r}[i] \in \mathbb{C}^{n_{t r} \times 1}$ are inputs at the source and at the relay, subject to the average power constraints $\frac{1}{n} \sum_{i=1}^{n}\|\boldsymbol{x}[i]\|^{2} \leq P_{s}$ and $\frac{1}{n} \sum_{i=1}^{n}\left\|\boldsymbol{x}_{r}[i]\right\|^{2} \leq P_{r}$, respectively; $\boldsymbol{y}_{1} \in \mathbb{C}^{n_{r 1} \times 1}, \boldsymbol{y}_{2} \in \mathbb{C}^{n_{r 2} \times 1}$ are the channel outputs at the respective user, while $\boldsymbol{y}_{r}[i] \in \mathbb{C}^{n_{r r} \times 1}$ stands for the relay output $y_{r}^{n} ; \boldsymbol{z}_{1}, \boldsymbol{z}_{2}$ and $\boldsymbol{z}_{r}$ are the additive Gaussian noises with temporarily and spatially i.i.d. entries $\mathcal{C N}(0,1)$; the set of all channel matrices $\boldsymbol{H}_{1}[i] \in \mathbb{C}^{n_{r 1} \times n_{t s}}$, $\boldsymbol{H}_{2}[i] \in \mathbb{C}^{n_{r 2} \times n_{t s}}, \boldsymbol{H}_{r}[i] \in \mathbb{C}^{n_{r r} \times n_{t s}}, \boldsymbol{G}_{1}[i] \in \mathbb{C}^{n_{r 1} \times n_{t r}}$, and $\boldsymbol{G}_{2}[i] \in \mathbb{C}^{n_{r 2} \times n_{t r}}$ are identified with the channel state $s_{i}=\left(\boldsymbol{H}_{1}[i], \boldsymbol{H}_{2}[i], \boldsymbol{H}_{r}[i], \boldsymbol{G}_{1}[i], \boldsymbol{G}_{2}[i]\right)$ and are temporarily i.i.d. channel matrix processes. In particular, $\boldsymbol{H}_{1}, \boldsymbol{H}_{2}$, and $\boldsymbol{H}_{r}$ are the channel matrices from the source to destination 1, destination 2, and the relay, respectively. Similarly, $\boldsymbol{G}_{1}$ and $G_{2}$ are matrices related to the relay-destination (RD) channels. Note that $n_{t s}$ and $n_{t r}$ denote the transmit antenna number at the source and the relay, respectively; $n_{r r}, n_{r 1}$, and $n_{r 2}$ are the number of the receive antennas at the relay, user 1 , and user 2 , respectively. Therefore, the joint distribution of the channel $p\left(y_{1}, y_{2}, y_{r} \mid x, x_{r}, s\right)$ in (1) can be specified as

$$
\begin{gathered}
p_{Z}\left(\boldsymbol{y}_{1}-\boldsymbol{H}_{1} \boldsymbol{x}-\boldsymbol{G}_{1} \boldsymbol{x}_{r}\right) p_{Z}\left(\boldsymbol{y}_{2}-\boldsymbol{H}_{2} \boldsymbol{x}-\boldsymbol{G}_{2} \boldsymbol{x}_{r}\right) p_{Z}\left(\boldsymbol{y}_{r}-\boldsymbol{H}_{r} \boldsymbol{x}_{r}\right) \\
=\frac{1}{\pi^{\prime}} e^{-\left\|\boldsymbol{y}_{1}-\boldsymbol{H}_{1} \boldsymbol{x}-\boldsymbol{G}_{1} \boldsymbol{x}_{r}\right\|^{2}-\left\|\boldsymbol{y}_{2}-\boldsymbol{H}_{2} \boldsymbol{x}-\boldsymbol{G}_{2} \boldsymbol{x}_{r}\right\|^{2}-\left\|\boldsymbol{y}_{r}-\boldsymbol{H}_{r} \boldsymbol{x}_{r}\right\|^{2}},
\end{gathered}
$$

where $\pi^{\prime}=\pi^{n_{r r}+n_{r 1}+n_{r 2}}$.

\section{Achievable Rate Regions for the Memoryless BRC}

In this section, we propose three achievable rate regions for the general two-user memoryless BRC. First, we provide the rate regions attained by the conventional decode-forward and compress-forward schemes, slightly adapted from the singleuser relay channel to the current setup. Then, we propose a decode-compress-forward (DCF) scheme that combines the relay observation and the state feedback in such a way that the destinations can benefit from the additional side information to decode the messages. An achievable rate region associated with the proposed scheme is presented.

\section{A. Conventional schemes}

In the following, we present two achievable rate regions using two conventional schemes. They are direct adaptation of the existing schemes from the single-user relay channel [1]. Therefore, the proofs are quite standard and omitted here. Proofs for similar settings can be found, e.g., in [2], [3], [20].

1) Decode-Forward (DF): Based on DF, the source transmits the signal $X$, that is encoded with $U_{1}$ and $U_{2}$ (depending on the messages), the relay decodes both messages, i.e. the indices of $U_{1}$ and $U_{2}$, from its observation $Y_{r}$, then re-encodes them and transmits $X_{1}, X_{2}$ via the encoded signal $X_{r}$ to the destinations. Hence, provided that the source messages are decoded correctly by the relay, the latter can cooperate with the source and form a virtual transmitter with additional resources (e.g., antennas, transmit power). An achievable region is shown below.

Proposition 1. (DF inner bound) An achievable rate region for the two-user memoryless state-dependent BRC by the DF scheme is given by the set of all rate pairs $\left(R_{1}, R_{2}\right)$ satisfying

$$
\begin{aligned}
& R_{1} \leq \min \left\{I\left(U_{1} X_{1} ; Y_{1} \mid S Q\right), I\left(U_{1} ; Y_{r} \mid X_{1} X_{2} U_{2} S Q\right)\right\}, \\
& R_{2} \leq \min \left\{I\left(U_{2} X_{2} ; Y_{2} \mid S Q\right), I\left(U_{2} ; Y_{r} \mid X_{1} X_{2} U_{1} S Q\right)\right\}, \\
& R_{1}+R_{2} \leq I\left(U_{1} U_{2} ; Y_{r} \mid X_{1} X_{2} S Q\right),
\end{aligned}
$$

for all pmf's that verify

$$
\begin{aligned}
& p\left(y_{1}, y_{2}, y_{r}, u_{1}, u_{2}, x_{1}, x_{2}, x, x_{r}, s, q\right)= \\
& p\left(y_{1}, y_{2}, y_{r} \mid x, x_{r}, s\right) p\left(x_{1}\right) p\left(x_{2}\right) p\left(u_{1} \mid x_{1}\right) p\left(u_{2} \mid x_{2}\right) \\
& \quad \times p\left(x \mid u_{1}, u_{2}, q\right) p\left(x_{r} \mid x_{1}, x_{2}, q\right) p(s) p(q) .
\end{aligned}
$$

The main ingredients of the above full-duplex DF scheme are the block-Markov superposition coding at the source 
and backward decoding at the destinations. Note that both transmitted signals at the source and the relay are independent of the instantaneous state of the channel due to the lack of CSIT. This feature will appear repeatedly also in the other schemes in this paper.

2) Compress-Forward (CF): Based on $\mathrm{CF}$, the transmitter sends the signal $X$, that is encoded with $U_{1}$ and $U_{2}$ (depending on the messages), the relay compresses the received signal $Y_{r}$, then encodes the description of the compressed signal $\hat{Y}$ and sends the encoded signal $X_{r}$ to the destinations. Hence, provided that the description of the compressed signal are decoded correctly at both destinations, the latter can cooperate with the relay and form virtual receivers with additional resources (e.g., antennas, received power). An achievable region is shown below.

Proposition 2. (CF inner bound) An achievable rate region for the two-user memoryless state-dependent BRC with the $C F$ scheme is given by the set of all rate pairs $\left(R_{1}, R_{2}\right)$ satisfying

$$
\begin{array}{r}
R_{1} \leq \min \left\{I\left(X_{r} U_{1} ; Y_{1} \mid S Q\right)-I\left(Y_{r} ; \hat{Y} \mid X_{r} U_{1} Y_{1} S Q\right),\right. \\
\left.I\left(U_{1} ; \hat{Y} Y_{1} \mid X_{r} S Q\right)\right\}, \\
R_{2} \leq \min \left\{I\left(X_{r} U_{2} ; Y_{2} \mid S Q\right)-I\left(Y_{r} ; \hat{Y} \mid X_{r} U_{2} Y_{2} S Q\right),\right. \\
\left.I\left(U_{2} ; \hat{Y} Y_{2} \mid X_{r} S Q\right)\right\},
\end{array}
$$

for all pmf's that verify

$$
\begin{aligned}
& p\left(y_{1}, y_{2}, y_{r}, \hat{y}, x_{r}, u_{1}, u_{2}, x, s, q\right)=p\left(y_{1}, y_{2}, y_{r} \mid x, x_{r}, s\right) \\
& \quad \times p\left(\hat{y} \mid x_{r}, y_{r}\right) p\left(u_{1}\right) p\left(u_{2}\right) p\left(x_{r}\right) p\left(x \mid u_{1}, u_{2}, q\right) p(s) p(q),
\end{aligned}
$$

subject to $I\left(Y_{r} ; \hat{Y} \mid X_{r} U_{k} Y_{k} S Q\right) \leq I\left(X_{r} ; Y_{k} \mid U_{k} S Q\right)$ for $k=$ 1,2 .

The main ingredients of the above full-duplex CF scheme are the block-Markov superposition coding at the source, Wyner-Ziv compression at the relay, and backward decoding at the destinations. Instead of decoding the original messages, the relay compresses the received signal, encodes the compression index, and then broadcasts the coded signal to the destinations.

\section{B. Decode-Compress-Forward (DCF) scheme}

We now present a rate region achieved by the proposed DCF scheme. The details of the scheme are rather technical and are deferred to the appendix. The essential idea is to let the relay forward some useful side information based on the decoded source messages and the state feedback from the destinations, in such a way that the interference can be mitigated in an efficient way. Specifically, the source first sends the encoded signal $X$, while the relay decodes both messages (the indices of $U_{1}$ and $U_{2}$ ) from observation $Y_{r}$ as in the DF scheme. Then, the relay compresses some function of the two decoded source messages and the CSI feedback from the destinations. Finally, the relay encodes the compression index (associated with $\hat{Y}$ ) with its own codebook and then transmits the encoded signal $X_{r}$ to both users as in the CF scheme. Each destination recovers the desired message jointly with the compression index. As in both the DF and CF schemes, the source and the relay transmits simultaneously with block-Markov coding. An achievable rate region is presented below.

Theorem 1. (DCF inner bound) An achievable region of memoryless state-dependent BRC with state feedback at the relay is given by the set of all rates pair $\left(R_{1}, R_{2}\right)$ satisfying

$$
\begin{aligned}
R_{1} & \leq \min \left\{I_{21}, I_{31}, I_{41}+I_{51}\right\}, \\
R_{2} & \leq \min \left\{I_{22}, I_{32}, I_{42}+I_{52}\right\}, \\
R_{1}+R_{2} & \leq I_{1},
\end{aligned}
$$

where, for $k \neq m \in\{1,2\}$,

$$
\begin{aligned}
I_{1} & \triangleq I\left(U_{1} U_{2} ; Y_{r} \mid X_{r} S Q\right), \\
I_{2 k} & \triangleq I\left(U_{k} ; Y_{r} \mid U_{m} X_{r} S Q\right), \\
I_{3 k} & \triangleq I\left(U_{k} ; Y_{k} \hat{Y} \mid X_{r} S Q\right), \\
I_{4 k} & \triangleq I\left(U_{k} ; Y_{k} \mid S Q\right), \\
I_{5 k} & \triangleq-I\left(\hat{Y} ; U_{m} \mid U_{k} X_{r} Y_{k} S Q\right)+I\left(X_{r} ; Y_{k} \mid U_{k} S Q\right),
\end{aligned}
$$

for all pmf's that verify $\min \left\{I_{51}, I_{52}\right\} \geq 0$ with

$$
\begin{aligned}
& p\left(y_{1}, y_{2}, y_{r}, \hat{y}, x, x_{r}, u_{1}, u_{2}, s, q\right)= \\
& p\left(\hat{y} \mid x_{r}, u_{1}, u_{2}, s, q\right) p\left(y_{1}, y_{2}, y_{r} \mid x, x_{r}, s\right) \\
& \quad \times p\left(x_{r} \mid q\right) p\left(x \mid u_{1}, u_{2}, q\right) p\left(u_{1}\right) p\left(u_{2}\right) p(s) p(q) .
\end{aligned}
$$

Sketch of proof: Without going into the details, the rate constraints can be interpreted as follows. First, the constraints $R_{1} \leq I_{21}, R_{2} \leq I_{22}$, and $R_{1}+R_{2} \leq I_{1}$ are the conditions required for the relay to recover both messages reliably. Then, $R_{k} \leq I_{3 k}$ is the condition for destination $k$ to decode the desired message with the help of the side information, provided that the latter is reliably delivered to destination $k$. Finally, the successful delivery of the side information at destination $k$ is guaranteed by the condition $R_{k} \leq I_{4 k}+I_{5 k}$. Details are deferred to the appendix.

In the conventional three-node relay network, decoding completely the messages and then compressing the recovered information is usually considered inefficient. Nevertheless, this is not the case in a multiple-user relay network, e.g., two user BRC with state feedback at the relay, since the main concern lies in how to reduce the interference. To mitigate the interference with our proposed scheme, the relay reconstructs the interference of each user based on the decoded messages and state feedback, compresses them and then forwards a description to the destinations. Furthermore, each receiver is capable of decoding the intended message with the side information about the corresponding interference. In contrast, we assume that the relay, rather than decode-compress-andforward, compresses directly its observation with the state feedback and then forwards to users. As a consequence, the relay creates a compression description of a mixture of the interference, instead of rebuilding the precise interference of each user. Thus, each user will receive undesired side information, which can be viewed as an 'interference' to the wanted side information.

Note that an achievable region for the broadcast channel (BC) with state feedback can be obtained straightforwardly from the above region, by removing the decoding constraints 
$I_{21}, I_{22}$, and $I_{1}$ at the relay. Then, we can recover the results in [15]. In addition, the convex hull of the achievable regions in [15] (on BC with causal state feedback at the transmitter) and in Proposition. 1 and 2 (by the adapted DF/CF schemes on BRC without state feedback) yields an achievable rate region of the broadcast relay channel with state feedback at the transmitter side. As no state feedback is provided at the relay, there is not much gain over DF/CF can be expected by simply exploiting the relay observation at the relay.

Moreover, it is worth mentioning that the presented rate regions for the general memoryless channel depend on the choice of pmf's involving the auxiliary random variables. Unfortunately, little intuition on the choice of "good" pmf's is provided from the mathematical expressions. As a result, it is in general extremely hard to evaluate the optimal rate region that includes all possible pmf choices. In the following, we shall focus on a special case, the fading Gaussian noise BRC, and propose particular choices on the pmf's, which allows us to evaluate the achievable rates.

\section{The Fading Gaussian Noise BRC}

For the fading Gaussian noise BRC described by (5), we consider two particular choices of the pmf's of the input variables $\left(X, X_{r}, U_{1}, U_{2}, \hat{Y}, Q\right)$. We regard the choices as different transmission schemes within the class of DCF, namely, the simultaneous emitted (SE) DCF and the time division (TD) DCF. We shall specify the chosen pmf's and the corresponding rate regions and then discuss the operational meaning of these choices.

\section{A. Simultaneous-Emitted Decode-Compress-Forward}

First, we present the SE DCF. The time-sharing RV $Q$ is binary with $P(Q=1)=\alpha_{1}$ and $P(Q=0)=1-\alpha_{1}$ with $\alpha_{1} \in[0,1]$. The triple $\left(X, X_{r}, \hat{Y}\right)$ is controlled by $Q$ as below

\begin{tabular}{|c|c|c|c|}
\hline & $X$ & $X_{r}$ & $\hat{Y}$ \\
\hline$Q=0$ & 0 & $\mathbf{x}_{r_{0}}$ & 0 \\
\hline$Q=1$ & $\mathbf{u}_{1}+\mathbf{u}_{2}$ & $\mathbf{x}_{r_{1}}$ & {$\left[\boldsymbol{H}_{1} \mathbf{u}_{2} \boldsymbol{H}_{2} \mathbf{u}_{1}\right]+\left[\hat{\mathbf{z}}_{1} \hat{\mathbf{z}}_{2}\right]$} \\
\hline
\end{tabular}

where $\mathbf{u}_{1} \sim \mathcal{C N}\left(\mathbf{0}, \boldsymbol{Q}_{1}\right), \mathbf{u}_{2} \sim \mathcal{C N}\left(\mathbf{0}, \boldsymbol{Q}_{2}\right), \mathbf{x}_{r_{1}} \sim \mathcal{C N}\left(\mathbf{0}, \boldsymbol{Q}_{r_{1}}\right)$ and $\mathbf{x}_{r_{0}} \sim \mathcal{C N}\left(\mathbf{0}, \boldsymbol{Q}_{r_{0}}\right)$, with $\alpha_{1}\left(\operatorname{tr}\left(\boldsymbol{Q}_{1}\right)+\operatorname{tr}\left(\boldsymbol{Q}_{2}\right)\right) \leq P_{s}$ and $\alpha_{1} \operatorname{tr}\left(\boldsymbol{Q}_{r_{1}}\right)+\left(1-\alpha_{1}\right) \operatorname{tr}\left(\boldsymbol{Q}_{r_{0}}\right) \leq P_{r}$. Intuitively, the signals $\mathbf{u}_{1}$ and $\mathbf{u}_{2}$ carrying two independent information flows are sent simultaneously through $X$. The interferences to both users are then compressed to $\hat{Y}$ with compression noises $\hat{\mathbf{z}}_{1} \sim \mathcal{C N}\left(\mathbf{0}, \boldsymbol{N}_{1}\right), \hat{\mathbf{z}}_{2} \sim \mathcal{C N}\left(\mathbf{0}, \boldsymbol{N}_{2}\right)$. The relay transmitted signal $\mathbf{x}_{r_{0}}$ or $\mathbf{x}_{r_{1}}$, known as the description of compression $\hat{Y}$, is identified with the auxiliary variable $X_{r}$ depending on the choice of $Q$.

With the above choices, the mutual information expressions involved in the rate region of Theorem 1 can be explicitly evaluated as (19)-(23).

Corollary 1 (SE-DCF Inner bound). For the fading Gaussian noise BRC with SD channel state feedback at the relay, a rate pair $\left(R_{1}, R_{2}\right)$ is achievable if (11), (12), (13), and $\min \left\{I_{51}, I_{52}\right\} \geq 0$ with the definitions (19)-(23) are satisfied for some $\boldsymbol{Q}_{1}, \boldsymbol{Q}_{2}, \boldsymbol{Q}_{r_{1}}, \boldsymbol{Q}_{r_{0}}, \boldsymbol{N}_{1}, \boldsymbol{N}_{2} \succeq 0$, and $\alpha_{1} \in[0,1]$ with $\alpha_{1}\left(\operatorname{tr}\left(\boldsymbol{Q}_{1}\right)+\operatorname{tr}\left(\boldsymbol{Q}_{2}\right)\right) \leq P_{s}$ and $\alpha_{1} \operatorname{tr}\left(\boldsymbol{Q}_{r_{1}}\right)+(1-$ $\left.\alpha_{1}\right) \operatorname{tr}\left(\boldsymbol{Q}_{r_{0}}\right) \leq P_{r}$.

$$
\begin{aligned}
& I_{1}=\alpha_{1} \mathbb{E} \log \operatorname{det}\left[\boldsymbol{I}+\boldsymbol{H}_{r}\left(\boldsymbol{Q}_{1}+\boldsymbol{Q}_{2}\right) \boldsymbol{H}_{r}^{H}\right] \\
& I_{2 k}=\alpha_{1} \mathbb{E} \log \operatorname{det}\left[\boldsymbol{I}+\boldsymbol{H}_{r} \boldsymbol{Q}_{k} \boldsymbol{H}_{r}^{H}\right] \\
& I_{3 k}=\alpha_{1} \mathbb{E} \log \operatorname{det}\left(\boldsymbol{I}+\boldsymbol{Q}_{k} \boldsymbol{H}_{k}^{H}\left(\boldsymbol{I}+\boldsymbol{H}_{k} \boldsymbol{Q}_{m} \boldsymbol{H}_{k}^{H}\left(\boldsymbol{H}_{k} \boldsymbol{Q}_{m} \boldsymbol{H}_{k}^{H}+\boldsymbol{N}_{k}\right)^{-1} \boldsymbol{N}_{k}\right)^{-1} \boldsymbol{H}_{k}+\boldsymbol{Q}_{k} \boldsymbol{H}_{m}^{H} \boldsymbol{N}_{m}^{-1} \boldsymbol{H}_{m}\right), \\
& \begin{aligned}
& I_{4 k}= \alpha_{1} \mathbb{E} \log \left[\frac{\operatorname{det}\left(\boldsymbol{I}+\boldsymbol{G}_{k} \boldsymbol{Q}_{r_{1}} \boldsymbol{G}_{k}^{H}+\boldsymbol{H}_{k}\left(\boldsymbol{Q}_{1}+\boldsymbol{Q}_{2}\right) \boldsymbol{H}_{k}^{H}\right)}{\operatorname{det}\left(\boldsymbol{I}+\boldsymbol{G}_{k} \boldsymbol{Q}_{r_{1}} \boldsymbol{G}_{k}^{H}+\boldsymbol{H}_{k} \boldsymbol{Q}_{m} \boldsymbol{H}_{k}^{H}\right)}\right] \\
& I_{5 k}=\alpha_{1} \mathbb{E} \log \left[\frac{\operatorname{det}\left(\boldsymbol{I}+\boldsymbol{G}_{k} \boldsymbol{Q}_{r_{1}} \boldsymbol{G}_{k}^{H}+\boldsymbol{H}_{k} \boldsymbol{Q}_{m} \boldsymbol{H}_{k}^{H}\right) \operatorname{det}\left(\boldsymbol{I}+\boldsymbol{H}_{k} \boldsymbol{Q}_{m} \boldsymbol{H}_{k}^{H}\right)}{\operatorname{det}\left(\boldsymbol{H}_{k} \boldsymbol{Q}_{m} \boldsymbol{H}_{k}^{H}+\boldsymbol{I}\right) \operatorname{det}\left(\boldsymbol{I}+\boldsymbol{H}_{k} \boldsymbol{Q}_{m} \boldsymbol{H}_{k}^{H}\left(\boldsymbol{I}+\boldsymbol{N}_{k}^{-1}\right)\right)}\right] \\
& \quad+\left(1-\alpha_{1}\right) \mathbb{E} \log \left[\operatorname{det}\left(\boldsymbol{I}+\boldsymbol{G}_{k} \boldsymbol{Q}_{r_{0}} \boldsymbol{G}_{k}^{H}\right)\right] .
\end{aligned}
\end{aligned}
$$

$$
\begin{aligned}
I_{1} & =\alpha_{1} \mathbb{E} \log \operatorname{det}\left[\boldsymbol{I}+\boldsymbol{H}_{r} \boldsymbol{Q}_{1} \boldsymbol{H}_{r}^{H}\right]+\alpha_{2} \mathbb{E} \log \operatorname{det}\left[\boldsymbol{I}+\boldsymbol{H}_{r} \boldsymbol{Q}_{2} \boldsymbol{H}_{r}^{H}\right] \\
I_{2 k} & =\alpha_{k} \mathbb{E} \log \operatorname{det}\left[\boldsymbol{I}+\boldsymbol{H}_{r} \boldsymbol{Q}_{k} \boldsymbol{H}_{r}^{H}\right] \\
I_{3 k} & =\alpha_{k} \mathbb{E} \log \operatorname{det}\left[\boldsymbol{I}+\boldsymbol{Q}_{k}\left(\boldsymbol{H}_{k}^{H} \boldsymbol{H}_{k}+\boldsymbol{H}_{m}^{H} \boldsymbol{N}_{k}^{-1} \boldsymbol{H}_{m}\right)\right] \\
I_{4 k} & =\alpha_{k} \mathbb{E} \log \left[\frac{\operatorname{det}\left(\boldsymbol{I}+\boldsymbol{H}_{k} \boldsymbol{Q}_{k} \boldsymbol{H}_{k}^{H}+\boldsymbol{G}_{k} \boldsymbol{Q}_{r_{k}} \boldsymbol{G}_{k}^{H}\right)}{\operatorname{det}\left(\boldsymbol{I}+\boldsymbol{G}_{k} \boldsymbol{Q}_{r_{k}} \boldsymbol{G}_{k}^{H}\right)}\right], \\
I_{5 k} & =\alpha_{k} \mathbb{E} \log \left[\frac{\operatorname{det}\left(\boldsymbol{I}+\boldsymbol{G}_{k} \boldsymbol{Q}_{r_{k}} \boldsymbol{G}_{k}^{H}\right)}{\operatorname{det}\left(\boldsymbol{I}+\boldsymbol{G}_{k} \boldsymbol{Q}_{r_{0}} \boldsymbol{G}_{k}^{H}\right)}\right]+\mathbb{E} \log \operatorname{det}\left(\boldsymbol{I}+\boldsymbol{G}_{k} \boldsymbol{Q}_{r_{0}} \boldsymbol{G}_{k}^{H}\right) \\
& \mathbb{E} \log \left[\frac{\operatorname{det}\left(\boldsymbol{I}+\boldsymbol{H}_{k} \boldsymbol{Q}_{m} \boldsymbol{H}_{k}^{H}+\boldsymbol{G}_{k} \boldsymbol{Q}_{r_{m}} \boldsymbol{G}_{k}^{H}\right)}{\operatorname{det}\left(\boldsymbol{I}+\boldsymbol{Q}_{m}\left(\boldsymbol{H}_{k}^{H}\left(\boldsymbol{I}+\boldsymbol{N}_{m}^{-1}\right) \boldsymbol{H}_{k}\right)\right) \operatorname{det}\left(\boldsymbol{I}+\boldsymbol{G}_{k} \boldsymbol{Q}_{r_{0}} \boldsymbol{G}_{k}^{H}\right)}\right] .
\end{aligned}
$$




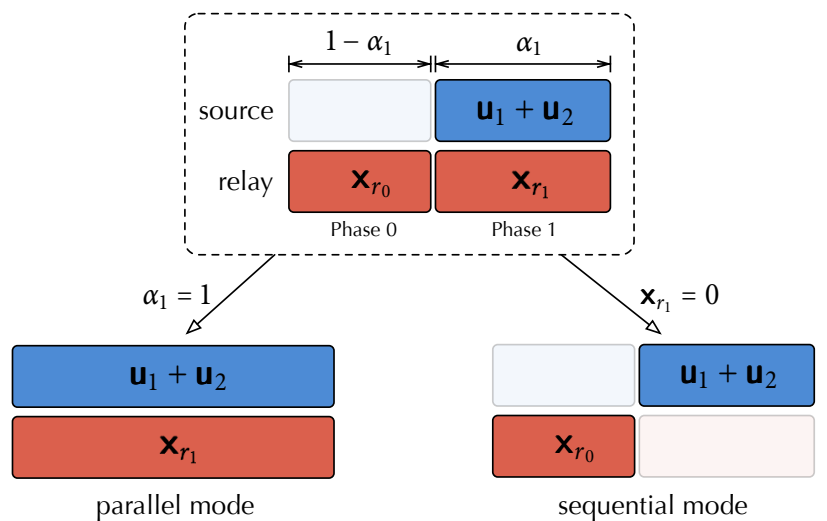

(a) Simultaneously emitted DCF

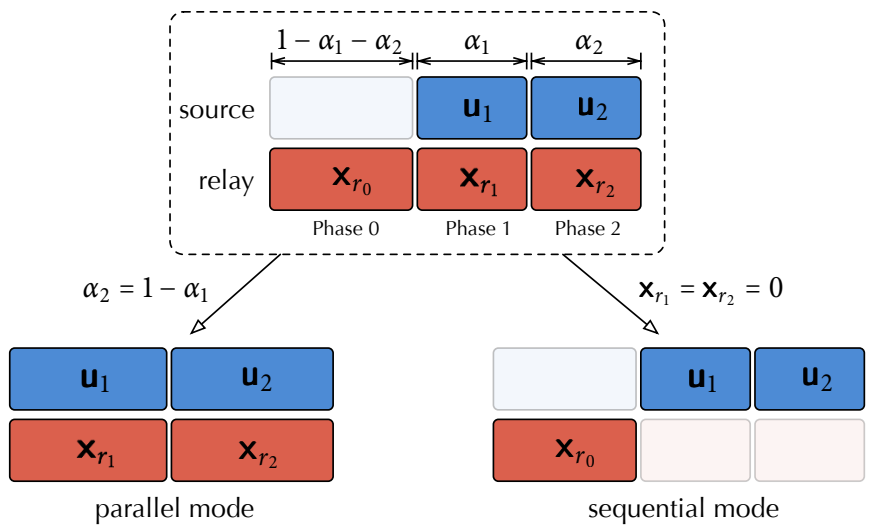

(b) Time-division DCF

Fig. 2. Block structure of the two DCF variants (SE-DCF and TD-DCF) in their general form and special modes.

\section{B. Time-Division Decode-Compress-Forward Scheme}

Next, we consider the TD DCF. The time-sharing RV $Q$ is ternary with $P(Q=1)=\alpha_{1}, P(Q=2)=\alpha_{2}$, and $P(Q=$ $0)=1-\alpha_{1}-\alpha_{2}$ with $\alpha_{1}, \alpha_{2}, \alpha_{1}+\alpha_{2} \in[0,1]$. The triple $\left(X, X_{r}, \hat{Y}\right)$ is controlled by $Q$ as below,

\begin{tabular}{|c|c|c|c|}
\hline & $X$ & $X_{r}$ & $\hat{Y}$ \\
\hline$Q=0$ & 0 & $\mathbf{x}_{r_{0}}$ & 0 \\
\hline$Q=1$ & $\mathbf{u}_{1}$ & $\mathbf{x}_{r_{1}}$ & $\boldsymbol{H}_{2} \mathbf{u}_{1}+\hat{\mathbf{z}}_{1}$ \\
\hline$Q=2$ & $\mathbf{u}_{2}$ & $\mathbf{x}_{r_{2}}$ & $\boldsymbol{H}_{1} \mathbf{u}_{2}+\hat{\mathbf{z}}_{2}$ \\
\hline
\end{tabular}

where $\mathbf{u}_{1} \sim \mathcal{C N}\left(\mathbf{0}, \boldsymbol{Q}_{1}\right), \mathbf{u}_{2} \sim \mathcal{C N}\left(\mathbf{0}, \boldsymbol{Q}_{2}\right), \mathbf{x}_{r_{q}} \sim \mathcal{C N}\left(\mathbf{0}, \boldsymbol{Q}_{r_{q}}\right)$, $q=0,1,2$, with $\alpha_{1} \operatorname{tr}\left(\boldsymbol{Q}_{1}\right)+\alpha_{2} \operatorname{tr}\left(\boldsymbol{Q}_{2}\right) \leq P_{s}$ and $\alpha_{1} \operatorname{tr}\left(\boldsymbol{Q}_{r_{1}}\right)+$ $\alpha_{2} \operatorname{tr}\left(\boldsymbol{Q}_{r_{2}}\right)+\left(1-\alpha_{1}-\alpha_{2}\right) \operatorname{tr}\left(\boldsymbol{Q}_{r_{0}}\right) \leq P_{r}$. Intuitively, the signals $\mathbf{u}_{1}$ and $\mathbf{u}_{2}$ carrying two independent information flows are sent successively through $X$. This is in contrast to the SE DCF in which the two flows are sent simultaneously. Each user overhears the other user's signal, i.e., user $k$ gets the noisy version of $\boldsymbol{H}_{k} \mathbf{u}_{j}$ when user $j$ 's signal is sent $(Q=j)$. The overheard signals are compressed to $\hat{Y}$ with compression noises $\hat{\mathbf{z}}_{1} \sim \mathcal{C N}\left(\mathbf{0}, \boldsymbol{N}_{1}\right), \hat{\mathbf{z}}_{2} \sim \mathcal{C N}\left(\mathbf{0}, \boldsymbol{N}_{2}\right)$. The relay transmitted signal $\mathbf{x}_{r_{0}}, \mathbf{x}_{r_{1}}, \mathbf{x}_{r_{2}}$, known as the description of compression $\hat{Y}$, is identified with the auxiliary variable $X_{r}$ depending on the choice of $Q$. With the above choices, the mutual information expressions involved in the rate region of Theorem 1 can be explicitly evaluated as (24)-(28).

Corollary 2. (TD DCF Inner bound) For the fading Gaussian noise BRC with $S D$ channel state feedback at the relay, a rate pair $\left(R_{1}, R_{2}\right)$ is achievable if (11), (12), (13), and $\min \left\{I_{51}, I_{52}\right\} \geq 0$ with the definitions (24)-(28) are satisfied for some $\boldsymbol{Q}_{1}, \boldsymbol{Q}_{2}, \boldsymbol{Q}_{r_{1}}, \boldsymbol{Q}_{r_{2}}, \boldsymbol{Q}_{r_{0}}, \boldsymbol{N}_{1}, \boldsymbol{N}_{2} \succeq 0$, and $\alpha_{1}, \alpha_{2}, \alpha_{1}+\alpha_{2} \in[0,1]$ with $\alpha_{1} \operatorname{tr}\left(\boldsymbol{Q}_{1}\right)+\alpha_{2} \operatorname{tr}\left(\boldsymbol{Q}_{2}\right) \leq P_{s}$ and $\alpha_{1} \operatorname{tr}\left(\boldsymbol{Q}_{r_{1}}\right)+\alpha_{2} \operatorname{tr}\left(\boldsymbol{Q}_{r_{2}}\right)+\left(1-\alpha_{1}-\alpha_{2}\right) \operatorname{tr}\left(\boldsymbol{Q}_{r_{0}}\right) \leq P_{r}$.

\section{Implementation of SE/TD DCF}

In the following, we explain the practical meaning of the probabilistic time-sharing variable $Q$. As will be shown later, each block can be structured in a deterministic way with $Q$ indicating the transmission phase within each block. As a result, the probability of $Q=q$ is related to the portion of the length of phase $q$. Therefore, we can implement SE DCF and TD DCF as is illustrated in Fig. 2. By tuning the parameters of the proposed DCF schemes, it is obvious that the performance in terms of throughput and complexity varies. Two extremes of the parameter configuration are the "parallel" mode and "sequential" mode. In the parallel mode, both the source and relay transmit during all the block, i.e., phase 0 is eliminated. In the sequential mode, the source and relay take turns to transmit without overlap. The sequential mode can thus be implemented even with half-duplex relays.

In both SE and TD DCF, the transmissions last for $B$ blocks. The structure of each block $b$ is given in Fig. 2. The relay transmits the encoded signal related to the side information for the previous block $b-1$. The encoded signal is $\left(x_{r_{0}}, x_{r_{1}}\right)$ in the SE-DCF case and $\left(x_{r_{0}}, x_{r_{1}}, x_{r_{2}}\right)$ in the TD-DCF case, as shown in Fig. 2(a) and Fig. 2(b) respectively. In phase 0 of both SE and TD DCF, the source remains silent. Then, the source transmits either the mixture of both information flows encoded in $u_{1}$ and $u_{2}$ in phase 1 (SE-DCF, Fig. 2(a)), or successively $u_{1}$ in phase 1 and $u_{2}$ in phase 2 (TD-DCF, Fig. 2(b)). In both cases, the relay listens and then decodes the source messages and generates the side information at the end of the block once the CSI feedback is available from the destinations.

\section{NUMERICAL RESUlts}

In order to assess the performance of the schemes proposed in Section IV, we investigate the sum-rate by numerical simulation in a two-user symmetric Gaussian fading BRC in presence of i.i.d. Rayleigh fading. In the following, we start by introducing the simulation parameters, and then compare the proposed schemes with conventional schemes, followed by some discussions.

\section{A. Simulation Parameters}

In the following numerical examples, we assume that $n_{t s}=$ $n_{t r}=n_{r r}=2$ and $n_{r 1}=n_{r 2}=1$. In other words, the source-destination and relay-destination channels are multipleinput single-output (MISO) channels, while the source-relay 


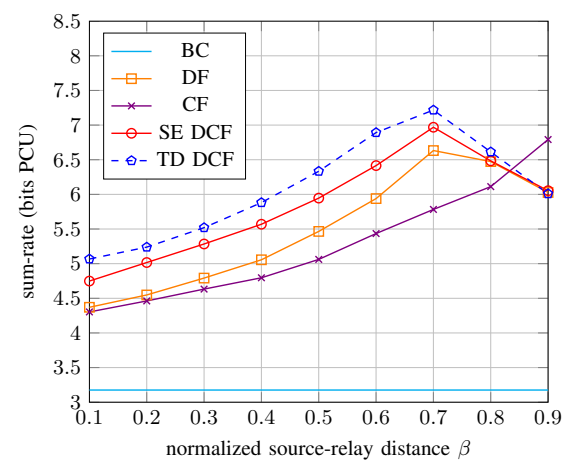

(a) $\mathrm{SNR}=10 \mathrm{~dB}$.

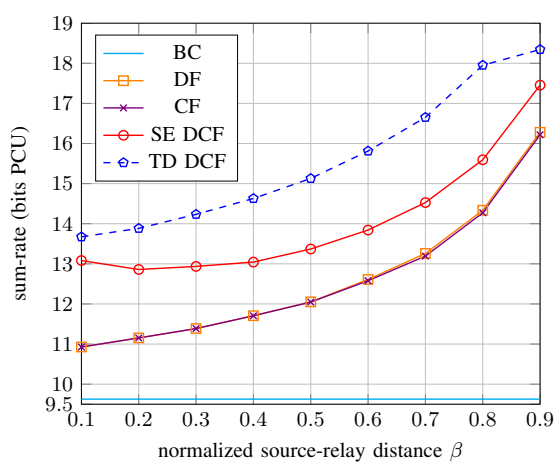

(b) $\mathrm{SNR}=30 \mathrm{~dB}$.

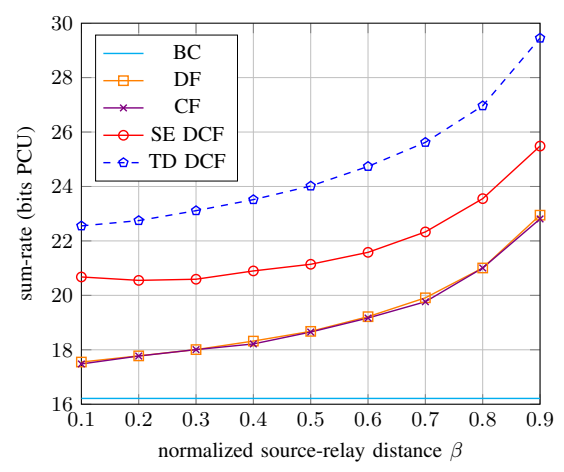

(c) $\mathrm{SNR}=50 \mathrm{~dB}$

Fig. 3. Sum-rate performance of the SE/TD DCF and the baseline schemes (BC/DF/CF) in different SNR regimes.

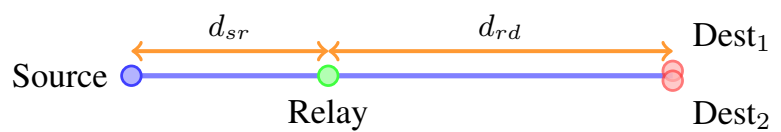

Fig. 4. The one-dimensional deployment of a two-user broadcast relay channel used in simulation.

channel is a multiple-input-multiple-output (MIMO) channel. This setup is motivated by the fact that both the macrocell and smallcell BS may have multiple antennas while it is generally harder for the terminals to have more than one antenna. In addition, we take into account a propagation model based only on the pathloss. In particular, pathloss is defined as $L=d^{-n_{p}}$, where $d$ denotes the distance between communication nodes and $n_{p}$ stands for the pathloss exponent. We let $n_{p}=2$, which corresponds to free-space propagation. $^{2}$ Therefore, the channel coefficients are rewritten as $L_{s d} \boldsymbol{H}_{k}, L_{s r} \boldsymbol{H}_{r}, L_{r d} \boldsymbol{G}_{k}$ for respective states of sourcedestination (SD), source-relay (SR), relay-destination (RD), with $L_{s d}, L_{s r}, L_{r d}$ being the pathloss for the channels and $\left(d_{s d}, d_{s r}, d_{r d}\right)$ the corresponding distances of (SD, SR, RD) channel. To simplify the evaluation, we adopt a onedimensional deployment as shown in Fig. 4, the source, the relay and the destinations are strictly aligned on a straight line where the distance between two users is neglected so that the two receivers are viewed as a single point in the deployment and yet have spatially i.i.d. channels. Hence, the distances between communication nodes are normalized as $\left(d_{s r}+d_{r d}\right) / d_{s d}=1$. We let $d_{s d}=10$ so that 9 integer-valued combinations of $\left(d_{s r}, d_{r d}\right)$ are picked. At both source and relay nodes, the same power constraints $\left(P_{s}=P_{r}=P\right)$ are taken. Since the noises are normalized, we define $\mathrm{SNR}=P L_{s d}$, which is the maximum SNR received at the users from the source.

In particular, in SE DCF, we let $\alpha_{1}=\alpha \in[0,1], \boldsymbol{Q}_{k}=$ $P_{k} \boldsymbol{I}_{2}, k=1,2$, and $\boldsymbol{Q}_{r_{q}}=P_{r_{q}} \boldsymbol{I}_{2}, q=0,1$. The power constraint in Corollary 1 implies that $\alpha\left(P_{1}+P_{2}\right) \leq P / 2$ and $(1-\alpha) P_{r_{0}}+\alpha P_{r_{1}} \leq P / 2$. In the TD DCF scheme, we equalize the duration of phase 1 and 2 as $\alpha_{1}=\alpha_{2}=\frac{1}{2} \alpha \in\left[0, \frac{1}{2}\right]$, set

\footnotetext{
${ }^{2}$ Other values of the pathloss exponent can be considered according to different propagation environments.
}

$\boldsymbol{Q}_{k}=P_{k} \boldsymbol{I}_{2}, k=1,2$, and $\boldsymbol{Q}_{r_{q}}=P_{r_{q}} \boldsymbol{I}_{2}, q=0,1,2$. The power constraint in Corollary 2 implies that $\alpha\left(P_{1}+P_{2}\right) \leq P$ and $2(1-\alpha) P_{r_{0}}+\alpha\left(P_{r_{1}}+P_{r_{2}}\right) \leq P$. Note that we optimize $\alpha$ and the power allocation individually in the simulations of all variants and modes.

In the simulations, we include three baseline schemes, namely, the broadcast channel (BC) without relay, the BRC with DF (Proposition 1) and the BRC with CF (Proposition 2) in the comparisons with SE/TD DCF. It is worth mentioning that only SE/TD DCF exploits state feedback while the reference schemes do not. Furthermore, all schemes are subject to the "no CSIT" constraint. We consider three levels of received SNR: the low-to-medium $(10 \mathrm{~dB})$, the medium-tohigh $(30 \mathrm{~dB})$ and the high level $(50 \mathrm{~dB})$. We focus on the sumrate performance in bits per channel use versus the normalized relay position $\beta=d_{s r} / d_{s d}$.

\section{B. DCF schemes versus the baseline schemes}

From Fig. 3(a), Fig. 3(b) and Fig. 3(c), the observations can be obtained followed by the discussion:

- Although not explicit in the figures, the first observation from the results is that each of the three baseline schemes achieves its respective optimal sum-rate with the strategy of the single-user transmission. Indeed, since no CSI is available at any of the transmitters, no gains are provided by serving more than one user at each transmission with the baseline schemes and no downlink multiplexing gain can be exploited.

- Beyond the medium SNR regime, all schemes perform better when the relay gets closer to the destinations, i.e., as $\beta$ increases. This observation indicates that the bottleneck of the current system lies in the relay-destination link. This is due to the fact that the multiplexing gain provided by the source-relay channel is higher than that of the other channels thanks to multiple antennas at the relay. This gain is not apparent at low-to-medium SNR.

- At low-to-medium SNR (SNR = 10dB), the sourcerelay link and the other links have similar quality at comparable distances. For both DF and DCF schemes, since the relay has to decode the source messages, there exists an optimal position for the relay between the source and the destinations, where the relay can reliably recover 


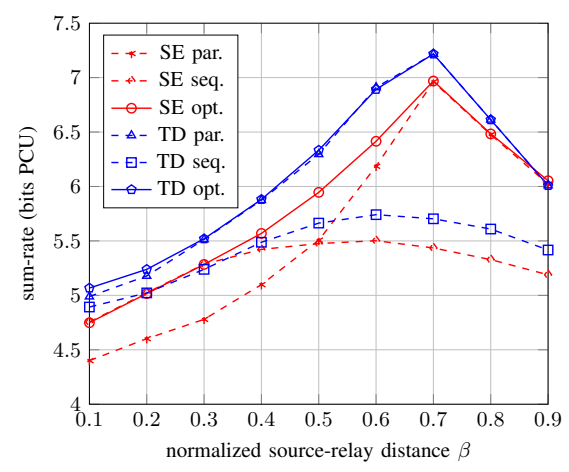

(a) $\mathrm{SNR}=10 \mathrm{~dB}$.

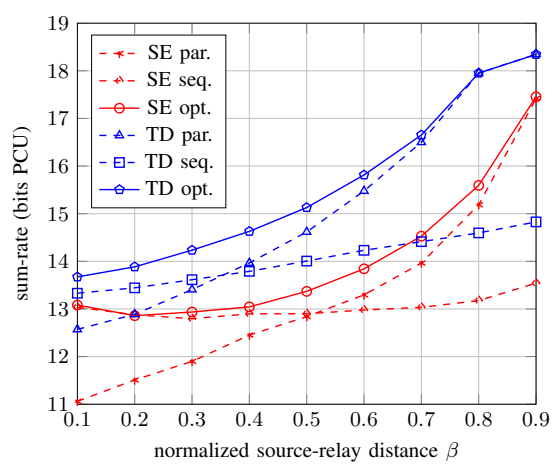

(b) $\mathrm{SNR}=30 \mathrm{~dB}$.

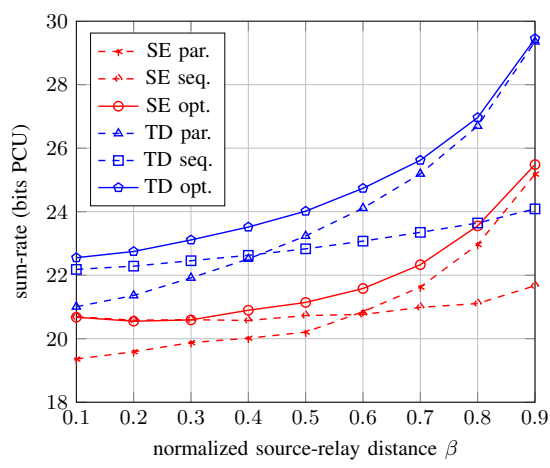

(c) $\mathrm{SNR}=50 \mathrm{~dB}$.

Fig. 5. Sum-rate performance of the variants with optimized parameters (“opt.”) and the simplified modes: parallel (“par.”) and sequential (“seq.”).

the messages and also help the destinations. This is to be contrasted with the high SNR regime in which the performance is limited by the source-destination links.

- In all regimes the TD DCF outperforms SE DCF and the performance gap increases with the SNR. Intuitively, this is due to the decoding constraint at the relay. Note that both streams are sent simultaneously with SE DCF, which requires the relay to decode both streams after a single transmission. With TD DCF, the relay can decode one stream at a time, which requires a lower source-relay link quality.

- Though it is not shown in the figure, the simulation results suggest that both the variants of the proposed scheme outperform, in terms of the sum-rate performance, the achievable region of broadcast channel with state feedback in [15] even when the transmitter is assigned with a $3 \mathrm{~dB}$ higher SNR in BC case, as long as the parameters in the proposed schemes' variants, i.e., $\alpha, \beta, P_{1}, P_{2}, P_{r_{0}}, P_{r_{1}}, P_{r_{2}}$, are properly chosen. ${ }^{3}$ Therefore, the proposed scheme has a sum-rate gain over the 'convex-hull' achievable region mentioned in III-B.

\section{DCF variants and "parallel/sequential" modes}

From Fig. 5(a), Fig. 5(b) and Fig. 5(c), we focus on variants of DCF schemes and the corresponding modes as follows:

- For both TD and SE DCF, the sequential mode presents a flat $\beta-R$ curve while parallel mode shows a sharp growth as the distance $\beta$ increases. In fact, in sequential mode, the optimal portion $\alpha$ varies with $\beta$. When the relay is close to the source, i.e., $\beta$ is small, $\alpha$ is large to compensate for a poor relay-destinations channel gain. And $\alpha$ decreases when $\beta$ increases. Unlike the sequential mode, in the parallel mode, the source and relay transmit concurrently. When $\beta$ is small, the relay signal is comparable to the source signal at the destinations, which means that the help of the relay comes at the cost of the source signal quality. The overall performance gain from the relay maybe marginal. When $\beta$ increases, the relay signal becomes stronger than the source signal, the

\footnotetext{
${ }^{3} \mathrm{We}$ assume that the relay power is assigned to the transmitter in $\mathrm{BC}$ case when comparing the sum-rate in $\mathrm{BC}$ case with that of $\mathrm{BRC}$, that is the SNR constraint at the transmitter in $\mathrm{BC}$ case is set to $13 \mathrm{~dB}, 33 \mathrm{~dB}, 53 \mathrm{~dB}$.
}

extra information provided by the relay can be obtained by the destinations without hurting the source signal. The overall performance gain becomes larger.

- When the relay is close to the source $(\beta \rightarrow 0)$, the sequential mode is near optimal, whereas the parallel model is near optimal when the relay is close to the destinations $(\beta \rightarrow 0){ }^{4}$ For general values of $\beta$, neither mode is close to the optimized curve. It implies that there exits a non-trivial value of $\alpha$ to fully exploit the presence of the relay, i.e., a hybrid version of orthogonal and nonorthogonal relaying is needed.

- Although it is not illustrated in the plots, we remark that an asymmetric power allocation $\left(P_{1} \neq P_{2}\right)$ can attain higher rate than symmetric case for SE DCF and the respective modes. On the one hand, extremely asymmetric power allocations are employed in $\mathrm{DF} / \mathrm{CF}$ scheme to mitigate the impact of interference and the absence of CSIT. On the other hand, the proposed scheme exploits space-time interference alignment and can cope with certain amount of interference. Therefore, asymmetric power allocation is exploited to alleviate the interference to a low level so that SE DCF can handle it.

\section{CONCLUSION}

In this work, we investigated on the downlink transmission of a single-cell multi-user heterogeneous system served by a source (macrocell BS) and a sub-tier relay (smallcell BS). We first provided a general achievable rate region by the proposed decode-compress-forward (DCF) scheme using state feedback from the destinations to the relay. In particular, we focused on two variants of the DCF scheme, namely, the time-division and simultaneously emitted DCF, in a fast fading Gaussian noise $\mathrm{BRC}$ and derived the respective achievable regions. To validate the theoretical results, we set up a one-dimensional deployment model and evaluate the sum-rate performance numerically. We found that our schemes can exploit the state feedback and provide a non-negligible performance gain as compared to the case where feedback is not used. We also revealed that the time-division DCF performs better than the

\footnotetext{
${ }^{4}$ optimal refers to the variants of the proposed scheme with optimized parameters, i.e., $\alpha, P_{r_{0}}, P_{r_{1}}, P_{r_{2}}$.
} 
simultaneously emitted DCF thanks to the low source-relay traffic load of the scheme.

\section{APPENDIX}

In this part, we present the proof of the achievability based on block-Markov superposition coding and random binning.

\section{Codebook generation:}

Given a pmf $p\left(\hat{y} \mid x_{r}, u_{1}, u_{2}, s, q\right) p\left(u_{1}\right) p\left(u_{2}\right) p\left(x \mid u_{1}, u_{2}, q\right)$ $p\left(x_{r} \mid q\right) p(s) p(q)$, the codebooks in each block are generated as follows.

1. Randomly generate $2^{n R_{k}}$ independent sequences $u_{k}^{n}$, which is drawn i.i.d. from $P_{U_{k}^{n}}\left(u_{k}^{n}\right)=\prod_{i=1}^{n} P_{U_{k}}\left(u_{k i}\right)$ and index them as $u_{k}^{n}\left(m_{k}\right)$ with $m_{k} \in\left\{1,2, \ldots, 2^{n R_{k}}\right\}$, $k=1,2$.

2. Generate randomly a sequence $q^{n}$, which is drawn i.i.d. from $P_{Q^{n}}\left(q^{n}\right)=\prod_{i=1}^{n} P_{Q}\left(q_{i}\right)$.

3 . For the generated sequence $q^{n}$, randomly and conditionally independently generate $2^{n R_{r}}$ independent sequences $x_{r}^{n}$ drawn i.i.d from $P_{X_{r}^{n} \mid Q^{n}}\left(x_{r}^{n} \mid q^{n}\right)=$ $\prod_{i=1}^{n} P_{X_{r} \mid Q}\left(x_{r i} \mid q_{i}\right)$ and index them as $x_{r}^{n}(r)$ with $r \in$ $\left\{1,2, \ldots, 2^{n R_{r}}\right\}$.

4. For each $x_{r}^{n}$ sequence, given the generated sequence $q^{n}$ and the state sequence $s^{n}$ feedback from receivers, randomly generate $2^{n \hat{R}}$ conditionally independent sequences $\hat{y}^{n}$ drawn i.i.d from $P_{\hat{Y}^{n} \mid X_{r}^{n}, S^{n}, Q^{n}}\left(\hat{y}^{n} \mid x_{r}^{n}, s^{n}, q^{n}\right)=$ $\prod_{i=1}^{n} P_{\hat{Y} \mid X_{r}, S, Q}\left(\hat{y}_{i} \mid x_{r, i}, s_{i}, q_{i}\right)$ and index them as $\hat{y}^{n}(l, r)$ with $l \in\left\{1,2, \ldots, 2^{n \hat{R}}\right\}$.

5. Partition the sets $\left\{l \mid l \in\left[1: 2^{n \hat{R}}\right]\right\}$ into $2^{n R_{r}}$ equal sized bins $\mathcal{B}(r)$ with $2^{n\left(\hat{R}-R_{r}\right)}$ elements in each bin, while $r \in\left[1: 2^{n R_{r}}\right]$ denotes the bin index.

6. Provide the codebooks and bins to the communication nodes.

\section{Encoding:}

The transmission is performed in $B+L$ blocks, where $B$ blocks are dedicated to convey the messages while the $L$ blocks assure the successful decoding of last index of auxiliary variable w.p. $1\left(L \rightarrow \infty, \frac{L}{B} \rightarrow 0\right)$.

Next, we focus on the source and the relay encoding as below.

- At each block $b \in[1: B]$, the transmitter communicates to each destination the messages $\left(m_{1 b}, m_{2 b}\right)$ based on $u_{1}^{n}\left(m_{1 b}\right), u_{2}^{n}\left(m_{2 b}\right)$ and time sharing random variable $q^{n}$. To be specific, encoder randomly picks and then sends $x^{n}(b)$ such that

$$
x^{n}(b) \in \mathcal{T}_{\delta}^{n}\left(X^{n}(b) \mid U_{1}^{n}\left(m_{1 b}\right), U_{2}^{n}\left(m_{2 b}\right), Q^{n}(b)\right) .
$$

For $b \in[B+1: B+L]$, the source transmits dummy messages with $m_{1 b}=m_{2 b}=1$.

- For each block $b=[1: B]$, provided that the relay receives the causal state feedback $s^{n}(b)$ and perfectly recovered the source messages $\left(m_{1 b}, m_{2 b}\right)$, the relay encoder searches for at least one index $l_{b}$ such that

$$
\begin{array}{r}
\left(u_{1}^{n}\left(m_{1 b}\right), u_{2}^{n}\left(m_{2 b}\right), \hat{y}^{n}\left(l_{b}, r_{b-1}\right), x_{r}^{n}\left(r_{b-1}\right), s^{n}(b), q^{n}(b)\right) \\
\in \mathcal{T}_{\delta}^{n}\left(U_{1}, U_{2}, \hat{Y}, X_{r}, S, Q\right) .
\end{array}
$$

The probability that such $l_{b}$ exists goes to one as $n$ tends to infinity if

$$
\hat{R} \geq I\left(\hat{Y} ; U_{1}, U_{2} \mid X_{r}, S, Q\right) .
$$

The relay obtains the bin index $r_{b}$ such that $l_{b} \in \mathcal{B}\left(r_{b}\right)$, which is sent at block $b+1$ in the form of $x_{r}^{n}\left(r_{b}\right)$. By convention, $r_{0}=1$. For $b=[B+1: B+L-1]$, at the block $b+1$, the relay repeats the last description of compression, namely $r_{B}$.

\section{Decoding and error events analysis:}

We assume that the sequence $q^{n}$ in each block is known to the transmitter, the relay, and both the receivers due to the fact that $q^{n}$ are chosen from the codebook according to the channel parameters/statistics and the transmitting protocol, where both are known to the communication nodes.

Then, the decoding strategies at the relay and the destinations are shown as below, followed by respective error events analysis.

1) A unique message pair $\left(m_{1 b}, m_{2 b}\right)$ is decoded at the relay once the source accomplishes its tranmissions at block $b$. The relay decoder searches for the unique $\left(m_{1 b}, m_{2 b}\right)$ such that

$$
\begin{array}{r}
\left(u_{1}^{n}\left(m_{1 b}\right), u_{2}^{n}\left(m_{2 b}\right), x_{r}^{n}\left(r_{b-1}\right), y_{r}^{n}(b), s^{n}(b), q^{n}(b)\right) \\
\in \mathcal{T}_{\delta}^{n}\left(U_{1}, U_{2}, X_{r}, Y_{r}, S, Q\right) .
\end{array}
$$

Thus, $\left(m_{1 b}, m_{2 b}\right)$ can be decoded with error probability goes to zero (as $n$ goes to infinity) provided that,

$$
\begin{aligned}
R_{1} & \leq I\left(U_{1} ; Y_{r} \mid X_{r} U_{2} S Q\right), \\
R_{2} & \leq I\left(U_{2} ; Y_{r} \mid X_{r} U_{1} S Q\right), \\
R_{1}+R_{2} & \leq I\left(U_{1} U_{2} ; Y_{r} \mid X_{r} S Q\right) .
\end{aligned}
$$

2) At every destination (let us take destination 1 as an example), $r_{B}$ is decoded at the end of block $B+L$ such that $\left(x_{r}^{n}\left(r_{B}\right), y_{1}^{n}(b), s^{n}(b), q^{n}(b)\right) \in \mathcal{T}_{\delta}^{n}\left(X_{r}, Y_{1}, S, Q\right)$ and the error probability goes to zero when $n$ tends to infinity, $b=[B+1: B+L]$ provided that,

$$
R_{r} \leq L \cdot I\left(X_{r} ; Y_{1} \mid S Q\right),
$$

which holds with high probability as $L$ goes to infinity $(L=O(B))$. With the assumption that $r_{B}$ be correctly recovered, a joint decoding is performed in a backward manner, i.e., for $b$ from $B$ to 1 , we find jointly $\left(\hat{r}_{b-1}, \hat{l}_{b}, \hat{m}_{1 b}\right)$ such that

$$
\begin{array}{r}
\left(u_{1}^{n}\left(\hat{m}_{1 b}\right), \hat{y}^{n}\left(\hat{l}_{b}, \hat{r}_{b-1}\right), x_{r}^{n}\left(\hat{r}_{b-1}\right), y_{1}^{n}(b), s^{n}(b), q^{n}(b)\right) \\
\in \mathcal{T}_{\delta}^{n}\left(U_{1} \hat{Y} X_{r} Y_{1} S Q\right) .
\end{array}
$$

The entire decoding procedure is designed backwardly, i.e., the indices $\left(\hat{r}_{b-1}, \hat{l}_{b}, \hat{m}_{1 b}\right)$ are decoded a priori, before the recovery of $\left(\hat{r}_{b-2}, \hat{l}_{b-1}, \hat{m}_{1(b-1)}\right)$ for $b \in$ $\{2,3, \cdots, B\}$.

The error events are analyzed as below.

- The probability of error event $\left(\hat{r}_{b-1}=r_{b-1}, \hat{l}_{b}=\right.$ $l_{b}, \hat{m}_{1 b} \neq m_{1 b}$ ) tends to zero if

$$
R_{1} \leq I\left(U_{1} ; Y_{1} \hat{Y} \mid X_{r} S Q\right) \text {. }
$$


- The error event such that $\left(\hat{r}_{b-1} \neq r_{b-1}, \hat{l}_{b}=\right.$ $\left.l_{b}, \hat{m}_{1 b}=m_{1 b}\right)$ could be avoided if

$$
\hat{R} \leq I\left(X_{r} \hat{Y} ; U_{1} Y_{1} \mid S Q\right) .
$$

- The probability of error event $\left(\hat{r}_{b-1}=r_{b-1}, \hat{l}_{b} \neq\right.$ $\left.l_{b}, \hat{m}_{1 b}=m_{1 b}\right)$ goes to zero if

$$
\hat{R}-R_{r} \leq I\left(\hat{Y} ; Y_{1} U_{1} \mid X_{r} S Q\right) .
$$

- The probability of error event $\left(\hat{r}_{b-1} \neq r_{b-1}, \hat{l}_{b}=\right.$ $\left.l_{b}, \hat{m}_{1 b} \neq m_{1 b}\right)$ tends to zero if

$$
R_{1}+\hat{R} \leq I\left(U_{1} X_{r} ; Y_{1} \mid S Q\right)+I\left(\hat{Y} ; Y_{1} U_{1} \mid X_{r} S Q\right) .
$$

- The probability of error event $\left(\hat{r}_{b-1}=r_{b-1}, \hat{l}_{b} \neq\right.$ $l_{b}, \hat{m}_{1 b} \neq m_{1 b}$ ) tends to zero if

$$
\begin{aligned}
& R_{1}+\hat{R}-R_{r} \leq \\
& \quad I\left(U_{1} ; Y_{1} \mid X_{r} S Q\right)+I\left(\hat{Y} ; Y_{1} U_{1} \mid X_{r} S Q\right) .
\end{aligned}
$$

- The probability of error event $\left(\hat{r}_{b-1} \neq r_{b-1}, \hat{l}_{b} \neq\right.$ $\left.l_{b}, \hat{m}_{1 b}=m_{1 b}\right)$ tends to zero if

$$
\hat{R} \leq I\left(X_{r} \hat{Y} ; Y_{1} U_{1} \mid S Q\right) .
$$

- The probability of error event $\left(\hat{r}_{b-1} \neq r_{b-1}, \hat{l}_{b} \neq\right.$ $\left.l_{b}, \hat{m}_{1 b} \neq m_{1 b}\right)$ tends to zero if

$$
R_{1}+\hat{R} \leq I\left(X_{r} U_{1} ; Y_{1} \mid S Q\right)+I\left(\hat{Y} ; Y_{1} U_{1} \mid X_{r} S Q\right) .
$$

3) Finally, with the same reasoning, we can obtain symmetric constraints for user 2 by exchanging indices in the constraints listed above. Moreover, the constraints (29)(32) and (34)-(40) are simplified by applying the FourierMotzkin elimination to $R_{r}$ and $\hat{R}$ successively. In the end, the redundant constraints are removed.

\section{REFERENCES}

[1] T. M. Cover and A. El Gamal, "Capacity Theorems for the Relay Channel," IEEE Trans. Inf. Theory, vol. 25, no. 5, pp. 572-584, Sep. 1979.

[2] G. Kramer, M. Gastpar, and P. Gupta, "Cooperative strategies and capacity theorems for relay networks," IEEE Trans. Inf. Theory, vol. 51, no. 9, pp. 3037-3063, 2005.

[3] A. Behboodi and P. Piantanida, "Cooperative strategies for simultaneous and broadcast relay channels," IEEE Trans. Inf. Theory, vol. 59, no. 3, pp. 1417-1443, 2013.

[4] L. Chen, "Parallel relay broadcast channels," in IEEE Int. Symp. Information Theory Proceedings (ISIT), 2010, pp. 585-589.

[5] G. Caire and S. Shamai, "On the achievable throughput of a multiantenna Gaussian broadcast channel," IEEE Trans. Inf. Theory, vol. 49, no. 7, pp. 1691-1706, 2003.

[6] H. Weingarten, Y. Steinberg, and S. Shamai, "The capacity region of the Gaussian multiple-input multiple-output broadcast channel," vol. 52, no. 9, pp. 3936-3964, 2006.

[7] C. Huang, S. A. Jafar, S. Shamai, and S. Vishwanath, "On degrees of freedom region of MIMO networks without channel state information at transmitters," IEEE Trans. Inf. Theory, vol. 58, no. 2, pp. 849-857, 2012.

[8] G. Caire, N. Jindal, M. Kobayashi, and N. Ravindran, "Multiuser MIMO achievable rates with downlink training and channel state feedback," IEEE Trans. Inf. Theory, vol. 56, no. 6, pp. 2845-2866, 2010.

[9] A. G. Davoodi and S. A. Jafar, "Aligned image sets under channel uncertainty: Settling a conjecture by Lapidoth, Shamai and Wigger on the collapse of degrees of freedom under finite precision CSIT," arXiv preprint arXiv:1403.1541, 2014.
[10] M. A. Maddah-Ali and D. Tse, "Completely stale transmitter channel state information is still very useful," IEEE Trans. Inf. Theory, vol. 58, no. 7, pp. 4418-4431, 2012.

[11] C. S. Vaze and M. K. Varanasi, "The degrees of freedom region and interference alignment for the MIMO interference channel with delayed CSIT," IEEE Trans. Inf. Theory, vol. 58, no. 7, pp. 4396-4417, 2012.

[12] R. Tandon, S. A. Jafar, S. Shamai Shitz, and H. V. Poor, "On the synergistic benefits of alternating CSIT for the MISO BC," IEEE Trans. Inf. Theory, vol. 59, no. 7, pp. 4106-4128, July. 2013.

[13] X. Yi, S. Yang, D. Gesbert, and M. Kobayashi, "The degrees of freedom region of temporally-correlated MIMO networks with delayed CSIT," IEEE Trans. Inf. Theory, vol. 60, no. 1, pp. 494 - 514, Jan. 2014.

[14] J. Chen and P. Elia, "Toward the performance versus feedback tradeoff for the two-user MISO broadcast channel," IEEE Trans. Inf. Theory, vol. 59, no. 12, pp. 8336-8356, Dec. 2013.

[15] C. He, S. Yang, and P. Piantanida, "On the capacity of the fading broadcast channel with state feedback," in IEEE Int. Symp. Communications, Control and Signal Processing (ISCCSP), May. 2014.

[16] — "An achievable rate region of broadcast relay channel with state feedback," in Proceedings of the IEEE International Conference on Communications (ICC), June. 2015, pp. 4193-4198.

[17] G. Atia and A. F. Molisch, "Cooperative relaying with imperfect channel state information," in IEEE Global Telecommunications Proceedings (GLOBECOM). IEEE, 2008, pp. 1-6.

[18] Y. Cui, V. K. Lau, and R. Wang, "Distributive subband allocation, power and rate control for relay-assisted OFDMA cellular system with imperfect system state knowledge," IEEE Trans. Wireless Communication, vol. 8, no. 10, pp. 5096-5102, 2009.

[19] W. Xu, X. Dong, and W.-S. Lu, "MIMO relaying broadcast channels with linear precoding and quantized channel state information feedback," IEEE Trans. Signal Processing, vol. 58, no. 10, pp. 5233-5245, 2010.

[20] A. El Gamal and Y.-H. Kim, Network Information Theory. Cambridge University Press, 2011. 